\title{
Increases in Acute Hepatitis B Virus Infections - Kentucky, Tennessee, and West Virginia, 2006-2013
}

Aaron M. Harris, MD ${ }^{1}$; Kashif Iqbal, MPH${ }^{1}$; Sarah Schillie, MD ${ }^{1}$; James Britton²; Marion A. Kainer, MBBS ${ }^{3}$; Stacy Tressler, MPH ${ }^{4}$; Claudia Vellozzi, MD ${ }^{1}$

As many as 2.2 million persons in the United States are chronically infected with hepatitis B virus (HBV) (1), and approximately $15 \%-25 \%$ of persons with chronic HBV infection will die prematurely from cirrhosis or liver cancer (2). Since 2006, the overall U.S. incidence of acute HBV infection has remained stable; the rate in 2013 was 1.0 case per 100,000 persons (3). Hepatitis $\mathrm{B}$ vaccination is highly effective in preventing $\mathrm{HBV}$ infection and is recommended for all infants (beginning at birth), all adolescents, and adults at risk for HBV infection (e.g., persons who inject drugs, men who have sexual contact with men, persons infected with human immunodeficiency virus [HIV], and others). Hepatitis $B$ vaccination coverage is low among adults: 2013 National Health Interview Survey data indicated that coverage with $\geq 3$ doses of hepatitis B vaccine was $32.6 \%$ for adults aged $19-49$ years (4). Injection drug use is a risk factor for both hepatitis $\mathrm{C}$ virus (HCV) and HBV. Among young adults in some rural U.S. communities, an increased incidence of HCV infection has been associated with a concurrent increase of injection drug use (5); and recent data indicate an increase of acute $\mathrm{HCV}$ infection in the Appalachian region associated with injection drug use (G). Using data from the National Notifiable Diseases Surveillance System (NNDSS) during 2006-2013, CDC assessed the incidence of acute HBV infection in three of the four Appalachian states (Kentucky, Tennessee, and West Virginia) included in the HCV infection study (6). Similar to the increase of HCV infections recently reported, an increase in incident cases of acute $\mathrm{HBV}$ infection in these three states has occurred among non-Hispanic whites (whites) aged 30-39 years who reported injection drug use as a common risk factor. Since 2009, cases of acute HBV infection have been reported from more nonurban than urban regions. Evidence-based services to prevent HBV infection are needed.

Data from confirmed cases of acute HBV infection reported to CDC from Kentucky, Tennessee, and West Virginia during 2006-2013, including demographic and risk characteristics, were obtained from NNDSS. These states used the CDC/ Council of State and Territorial Epidemiologists case definition to identify cases of acute $\mathrm{HBV}$ infection. ${ }^{\dagger}$ Cases of acute $\mathrm{HBV}$ infection were categorized as "urban" if the infected person

\footnotetext{
${ }^{\dagger}$ A person with acute illness with a discrete onset of symptoms and either jaundice or elevated serum alanine aminotransaminase levels and a positive test result for immunoglobulin $\mathrm{M}$ antibody to hepatitis $\mathrm{B}$ core antigen and hepatitis B surface antigen (http://wwwn.cdc.gov/nndss/conditions/ hepatitis-b-acute/).
}

lived in a metropolitan county with a population $\geq 50,000$ and as "non-urban" if the infected person lived in a nonmetropolitan county with a population $<50,000 .{ }^{\S}$ Data were analyzed by year of report and urban/non-urban county resident status to assess annual incidence (per 100,000 persons), demographic characteristics, and injection drug use in persons with reported acute HBV infections during 2006-2013. To calculate annual incidence, the number of cases reported through NNDSS was used as the numerator and midyear (July) population estimates from the U.S. Census Bureau were used as the denominator. Statistical significance of a monotonic trend in annual incidence of acute HBV infection by urban/non-urban status was tested with the Spearman rank correlation test. A 20\% increase in incident $\mathrm{HBV}$ infections was observed from 2009 to 2010; therefore, the data are presented for two reporting time periods: 2006-2009 and 2010-2013. Chi-square tests were used to determine whether cases reported during the two time periods differed significantly by demographic characteristics and reported injection drug use. Statistical significance was defined as $\mathrm{p}<0.05$.

During 2006-2013, a total of 3,305 cases of acute HBV infection were reported to CDC from Kentucky, Tennessee, and West Virginia. During 2009-2013, incidence of acute HBV infection increased $114 \%$ in these three states, but remained stable in the United States overall (Figure 1). Comparing the number of cases of acute HBV infection reported during 2006 2009 and 2010-2013, the proportion of cases among whites and persons aged 30-39 years increased during 2010-2013 (Table). Among cases in which at least one risk factor was reported, the proportion of persons reporting injection drug use as a risk factor was significantly greater in 2010-2013, compared with 2006-2009 (75\% versus 53\%; p<0.001).

Among 3,185 of 3,305 (96\%) total cases where urban and non-urban classification for HBV-infected persons could be determined, 1,344 (42\%) were classified as residing in nonurban counties. During 2006-2013, the incidence of acute HBV infections from both urban and non-urban counties increased, but the increase was statistically significant only among cases occurring in non-urban counties (Figure 2) (p-value for trend $<0.001)$.

\$ http://www.cdc.gov/nchs/data/series/sr_02/sr02_166.pdf. 


\section{Summary}

What is already known on this topic?

The national incidence of hepatitis B virus (HBV) infection has remained stable during 2006-2013 at 1 case per 100,000 persons. Currently, as many as 2.2 million persons are chronically infected with HBV. Injection drug use is a risk factor for HBV transmission.

What is added by this report?

Since 2009, three states in the Appalachian region have reported an increase in cases of acute HBV infection, among non-Hispanic whites, persons aged $30-39$ years, and injection drug users. Compared with cases that occurred during 20062009, a significant increase in the proportion of cases in which injection drug use was reported during 2010-2013.

What are the implications for public health practice?

The increase in incident HBV-infections has the potential to impede the nation's hepatitis B elimination strategy. Evidencebased prevention strategies, including increasing hepatitis $B$ vaccination coverage, testing and linkage to care, and implementing education campaigns that target persons who inject drugs are urgently needed.

FIGURE 1. Incidence of acute hepatitis B virus infection, by yearUnited States and Kentucky, Tennessee, and West Virginia, 2006-2013

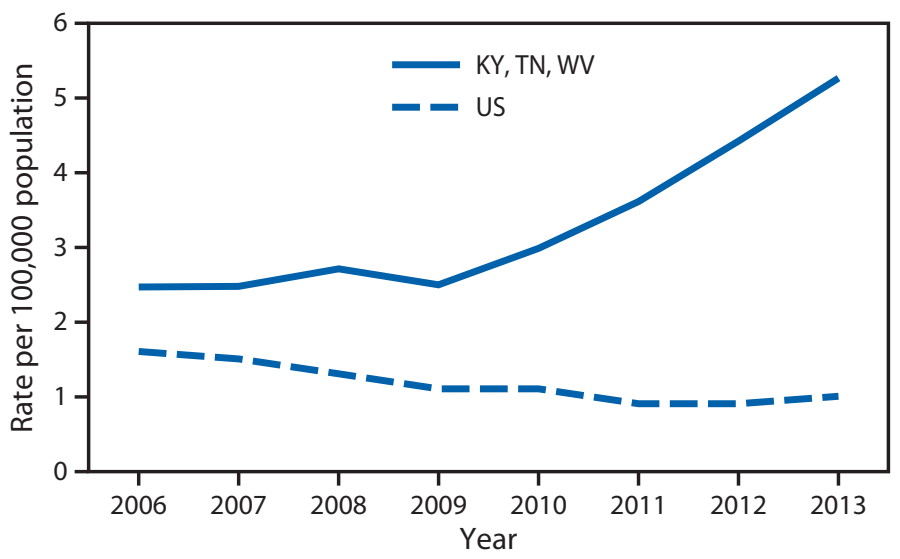

Abbreviations: $\mathrm{KY}=$ Kentucky; TN = Tennessee; US = United States; WV = West Virginia..

\section{Discussion}

Population-based surveillance data from Kentucky, Tennessee, and West Virginia indicate a $114 \%$ increase in acute HBV infection during 2006-2013; this increase occurred after 2009, among whites, aged 30-39 years who reported injection drug use. In an analysis of 6 years of enhanced surveillance data for hepatitis B, Tennessee reported similar findings, including a large increase among white adults, with both injection and noninjection drug use as a commonly reported risk factor during 2006-2011 (7).
TABLE. Demographic characteristics and injection drug use behavior for 3,305 reported cases of acute hepatitis B virus infection, by reporting period - Kentucky, Tennessee, and West Virginia, 2006-2013

\begin{tabular}{|c|c|c|c|}
\hline \multirow[b]{2}{*}{ Characteristic* } & \multicolumn{2}{|c|}{ Reporting period } & \multirow[b]{2}{*}{$\mathrm{p}$-value } \\
\hline & $\begin{array}{c}2006-2009 \\
(n=1,243) \\
\text { No. }(\%)\end{array}$ & $\begin{array}{c}2010-2013 \\
(\mathrm{n}=2,062) \\
\text { No. }(\%)\end{array}$ & \\
\hline Age group (yrs) & & & $<0.001$ \\
\hline $0-18$ & $6(0.5)$ & $7(0.3)$ & \\
\hline $19-29$ & $290(23.3)$ & $371(18.0)$ & \\
\hline $30-39$ & $354(28.5)$ & $763(37.0)$ & \\
\hline $40-49$ & $356(28.7)$ & $537(26.0)$ & \\
\hline $50-59$ & $153(12.3)$ & $254(12.3)$ & \\
\hline$\geq 60$ & $83(6.7)$ & $128(6.2)$ & \\
\hline Sex & & & 0.582 \\
\hline Male & $736(59.4)$ & $1,196(58.4)$ & \\
\hline Female & $503(40.6)$ & $851(41.6)$ & \\
\hline Race & & & $<0.001$ \\
\hline Non-Hispanic black & $128(12.5)$ & $128(7.6)$ & \\
\hline Non-Hispanic white & 869 (84.6) & $1,503(88.9)$ & \\
\hline Hispanic & $13(1.3)$ & $20(1.2)$ & \\
\hline Other & $17(1.7)$ & $39(2.3)$ & \\
\hline Injection drug use ${ }^{\dagger}$ & & & $<0.001$ \\
\hline Yes & $180(52.9)$ & $342(75.2)$ & \\
\hline No & $160(47.1)$ & $113(24.8)$ & \\
\hline
\end{tabular}

* Percentage among patients with a valid response; unknown and missing not included.

+ Among patients with at least one reported risk factor on the data abstraction form; $\mathrm{N}=795$ (2006-2009: $\mathrm{n}=340 ; 2010-2013: \mathrm{n}=455)$; unknown and missing not included.

Forty-two percent of cases of acute HBV infection in this report occurred among persons residing in non-urban counties, which is where the largest increases in incidence of acute HBV infection occurred. A similar increase of acute HCV infections occurred among young adults residing in non-urban areas in Kentucky, Tennessee, Virginia, and West Virginia (G). The concurrent increase in reports of acute HBV and HCV infections, as well as an increase in injection drug use reported among this population is concerning. Together, the increase in cases of acute $\mathrm{HBV}$ infection among persons who reported injection drug use and the typically low hepatitis $\mathrm{B}$ vaccination coverage among young adults are likely contributing to the increase in acute HBV infection incidence in Kentucky, Tennessee, and West Virginia. A concomitant increase in the number of substance abuse treatment admissions for opioid dependency in Appalachian states during 2006-2013 was also observed: admissions for prescription opioid and heroin abuse increased among young adults by $17.1 \%$ and $7.4 \%$, respectively (6). In 2015, a rural county in Indiana was the site of a large outbreak of HIV infection and HCV infection among young (median age $=32$ years) injection drug users $(8)$.

Hepatitis $B$ vaccination is recommended as primary prevention for adults who are at increased risk for HBV infection, 
FIGURE 2. Incidence* of acute hepatitis B virus infection by urban/non-urban ${ }^{\dagger}$ county of residence - Kentucky, Tennessee, and West Virginia, 2006-2013

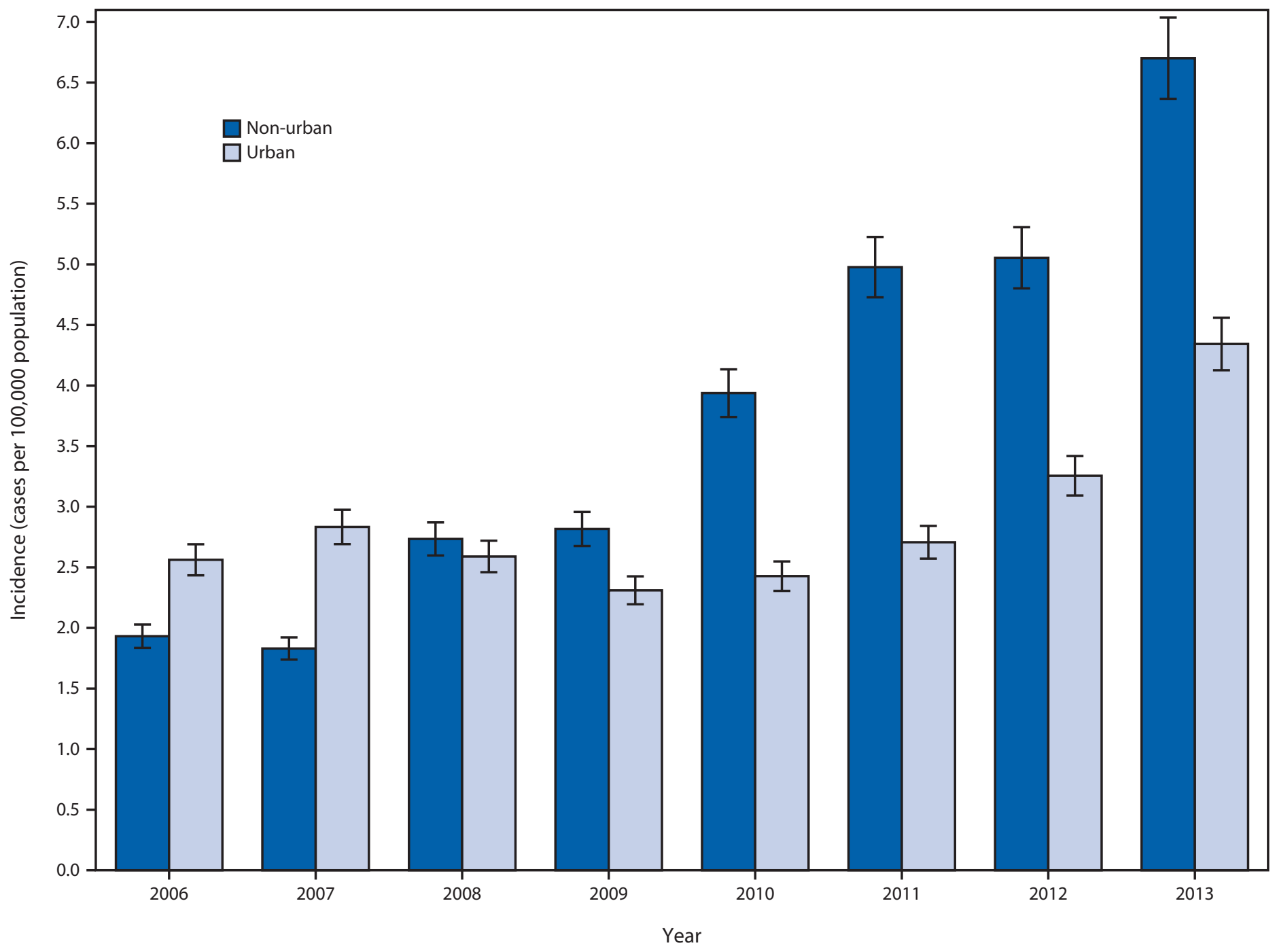

* With $95 \%$ confidence intervals as error bars.

† Trend significant among non-urban residence data at $\mathrm{p}<0.001$.

including injection drug users who were not previously infected (9). Data from the National Health Interview Survey indicate that hepatitis $\mathrm{B}$ vaccination coverage is low among adults in the general population (4), and it is likely to be lower among injection drug users. Routine hepatitis B vaccination has been recommended for infants since 1991 and for children aged $\leq 18$ years since 1999; thus, adults aged $\geq 33$ years in 2013 would be too old to have benefited from routine hepatitis $B$ vaccination recommendations, and would be susceptible to $\mathrm{HBV}$ infection.

In response to this increase in acute HBV infections, state health officials are employing various prevention strategies. Since 2012, Tennessee has partnered with county jails to increase hepatitis B vaccination coverage among incarcerated persons. West Virginia has collaborated with addiction centers and harm reduction services to provide viral hepatitis prevention trainings. West Virginia is establishing an adult hepatitis $\mathrm{B}$ vaccination pilot project in the 17 counties with the highest incidence of acute HBV infection. To enhance viral hepatitis surveillance in Kentucky, reporting of HBV infection among pregnant women and children aged $<5$ years, in addition to all acute HBV infection cases, is mandatory. Kentucky has also increased hepatitis B awareness campaigns through annual statewide hepatitis conferences, health care provider education, and legislative amendments allowing syringe exchange programs.

The National Viral Hepatitis Action Plan recommends full vaccination of adolescents, as well as ensuring that injection drug users have access to viral hepatitis prevention, care, and 
treatment services (10). This can be accomplished by mobilizing community resources to identify persons at risk, increase hepatitis $\mathrm{B}$ vaccination coverage among all adolescents and adult injection drug users, screen and test for HBV, HCV, and HIV infections, and link persons with viral hepatitis to care. A goal for hepatitis B elimination is vaccination of all vulnerable youth and adults; thus, the delivery of hepatitis prevention and care should be expanded to include correctional facilities and abuse treatment centers.

The findings in this report are subject to at least five limitations. First, NNDSS is a passive surveillance system, and therefore, unreported cases might have been missed. Second, the current case definition for acute HBV infection captures only symptomatic persons and excludes persons with asymptomatic $\mathrm{HBV}$ infection, and therefore might result in underreporting of total acute HBV cases. Third, acute HBV infection case reports typically originate from past or present medical care; thus, certain populations at high risk (e.g., persons who are incarcerated, homeless, and uninsured) with limited access to care could potentially be underrepresented. Fourth, increased reporting and changes in testing practices might have contributed to the increase in HBV incidence observed in the three Appalachian states in this report. However, an upward trend in incidence was not seen in other areas of the country, and began before the release of the CDC HCV testing recommendations that might have affected HBV testing and reporting. Finally, risk factor data, including injection drug use, were not available for all reported cases.

A hepatitis B epidemic is emerging in Kentucky, Tennessee, and West Virginia. The increase in incident HBV-infections might contribute to future increases in liver-related morbidity and mortality. Evidence-based prevention strategies, including increasing hepatitis $B$ vaccination coverage, testing and linkage to care activities, and education campaigns targeting persons who inject drugs are urgently needed.
${ }^{1}$ Division of Viral Hepatitis, CDC; ${ }^{2}$ Kentucky Department for Public Health; ${ }^{3}$ Tennessee Department of Health; ${ }^{4}$ West Virginia Department of Health and Human Services.

Corresponding author: Aaron M. Harris, AMHarris@cdc.gov, 404-718-8541.

\section{References}

1. Kowdley KV, Wang CC, Welch S, Roberts H, Brosgart CL. Prevalence of chronic hepatitis B among foreign-born persons living in the United States by country of origin. Hepatology 2012;56:422-33. http://dx.doi. org/10.1002/hep.24804.

2. Fattovich G, Bortolotti F, Donato F. Natural history of chronic hepatitis B: special emphasis on disease progression and prognostic factors. J Hepatol 2008;48:335-52. http://dx.doi.org/10.1016/j. jhep.2007.11.011.

3. CDC. Viral hepatitis statistics and surveillance. Surveillance for viral hepatitis-United States, 2013. Atlanta, GA: US Department of Health and Human Services; 2015. http://www.cdc.gov/hepatitis/ statistics/2013surveillance/index.htm.

4. Williams WW, Lu PJ, O'Halloran A, et al. Noninfluenza vaccination coverage among adults-United States, 2013. MMWR Morb Mortal Wkly Rep 2015;64:95-102.

5. Christian WJ, Hopenhayn C, Christian A, McIntosh D, Koch A. Viral hepatitis and injection drug use in Appalachian Kentucky: a survey of rural health department clients. Public Health Rep 2010;125:121-8.

6. Zibbell JE, Iqbal K, Patel RC, et al. Increases in hepatitis $\mathrm{C}$ virus infection related to injection drug use among persons aged $\leq 30$ yearsKentucky, Tennessee, Virginia, and West Virginia, 2006-2012. MMWR Morb Mortal Wkly Rep 2015;64:453-8.

7. Iqbal K, Klevens RM, Kainer MA, et al. Epidemiology of acute hepatitis B in the United States from population-based surveillance, 2006-2011. Clin Infect Dis 2015;61:584-92. http://dx.doi.org/10.1093/cid/civ332.

8. Conrad C, Bradley HM, Broz D, et al. Community outbreak of HIV infection linked to injection drug use of oxymorphone-Indiana, 2015. MMWR Morb Mortal Wkly Rep 2015;64:443-4.

9. Mast EE, Margolis HS, Fiore AE, et al. A comprehensive immunization strategy to eliminate transmission of hepatitis B virus infection in the United States: recommendations of the Advisory Committee on Immunization Practices (ACIP) part 1: immunization of infants, children, and adolescents. MMWR Recomm Rep 2005;54(No. RR-16).

10. US Department of Health and Human Services. Action plan for the prevention, care, and treatment of viral hepatitis. Washington, DC: US Department of Health and Human Services; 2014. https://www.aids. gov/pdf/viral-hepatitis-action-plan.pdf. 